\title{
Relationship between Cognitive Function, Depression/Anxiety and Functional Parameters in Patients Admitted for Congestive Heart Failure
} \author{
and Marzia Testa ${ }^{4}$ \\ ${ }^{1}$ Cardiovascular Rehabilitation-Heart Failure Unit Ospedale SS Trinita' Fossano, Italy \\ ${ }^{2}$ Psychology Service ASL CN1 Cuneo, Italy \\ ${ }^{3}$ Cardiology Division Ospedale S Croce-Carle Cuneo, Italy \\ ${ }^{4}$ School of Geriatry Universita' degli Studi Torino, Italy
}

Mauro Feola ${ }^{1, *}$, Sonia Garnero ${ }^{1}$, Paola Vallauri ${ }^{2}$, Luigi Salvatico ${ }^{2}$, Antonello Vado ${ }^{3}$, Laura Leto ${ }^{4}$

Abstract: Cognitive impairment, anxiety and depression have been described in patients with congestive heart failure (CHF). We analyzed in-hospital CHF patients before discharge with neuropsychological tests attempting to correlate with prognostic parameters.

Methods: All subjects underwent a mini mental state examination (MMSE), geriatric depression scale (GDS), anxiety and depression scale test (HADS). We evaluated NYHA class, brain natriuretic peptide (BNP), left ventricular ejection fraction (LVEF) and non-invasive cardiac output (CO).

Results: Three-hundred and three CHF patients (age 71.6 ys) were analysed. The mean NYHA class was $2.9 \pm 0.8$, LVEF was $43.4 \pm 15.8 \%$; BNP plasma level and $\mathrm{CO}$ were calculated as $579.8 \pm 688.4 \mathrm{pg} / \mathrm{ml}$ and $3.9 \pm 1.1 \mathrm{l} / \mathrm{min}$, respectively. In 9.6\% a pathological MMSE score emerged; a depression of mood in $18.2 \%$ and anxiety in $23.4 \%$ of patients were observed. A significant correlation between MMSE and age $(\mathrm{r}=0.11 \mathrm{p}=0.001), \mathrm{BNP}(\mathrm{r}=0.64 \mathrm{p}=0.03)$ but not between MMSE and NYHA class and LVEF was observed. GDS and HADS were inversely correlated with NYHA class $(\mathrm{r}=0.38 \mathrm{p}=0.04)$ and six-minute walking test $(\mathrm{r}=0.18 \mathrm{p}=0.01)$ without an association with objective parameters in CHF (BNP, LVEF and cardiac output). At multivariate analysis only MMSE and BNP are inversely correlated significantly ( $\mathrm{p}=0.019 \mathrm{OR}=-0.64$, $\mathrm{CI}=-042-0.86)$.

Conclusions: in-hospital CHF patients may manifest a reduction of MMSE and important anxiety/depression disorders. The results of the study suggest that the presence of cognitive impairment in older CHF patients with higher BNP plasma level should be considered. In admitted CHF patients anxiety and depression of mood are commonly reported and influenced the perception of the severity of illness.

Keywords: Cognitive impairment, heart failure, brain natriuretic peptide.

\section{INTRODUCTION}

Chronic heart failure is a major health problem, which is growing together with the increasingly age of population. Recurrent hospitalisations occur in about half of the subjects within 6 months after the initial admission [1]. Several comorbidities usually coexist in these patients [2]. In spite of its clinical importance, the relationship between cognitive impairment and clinical status in chronic heart failure patients has not always been considered in studies and in clinical interventions regarding patients with congestive heart failure (CHF) [3-5]. Recently even in the early stage of left

\footnotetext{
*Address correspondence to this author at the Cardiovascular Rehabilitation-Heart Failure Unit, Ospedale SS Trinita' Via Ospedale 412045 Fossano Italy; Tel: +390172699365; Fax: +39-0172699364;

E-mail:m_feola@virgilio.it
}

ventricular dysfunction and heart failure a worse cognitive functioning can be observed [6] and that plasma Brain Natriuretic Peptide (BNP) correlated inversely with attention and functioning. Moreover, in $\mathrm{CHF}$ patients a variable prevalence of depression of mood (20-41.8\% according to the different definitions or setting of evaluation) $[7,8]$ or anxiety $(25 \%)$ have been described.

The aim of this observational study was to analyse the prevalence of cognitive impairment and anxiety/depression an in-hospital CHF population trying to correlate with functional parameters of illness severity collected.

\section{METHODS}

From April 2008 to June 2011, all consecutive CHF patients admitted to our Department for new diagnosis of CHF 
or for acute decompensation in chronic CHF were requested to enter the study. Subjects of any age, aetiology, and severity of symptoms were recruited. All patients underwent a complete medical, psychological and neuropsychological examination. Patients included in this study fulfilled all (1-3) the following criteria:

1. symptoms of CHF, according to the criteria commonly accepted in medical literature [9], namely the presence of 2 major criteria or 1 major criterion +2 minor criteria according to the Framingham score (dyspnoea on exertion, orthopnea, paroxysmal nocturnal dyspnoea, lower extremity oedema, rapid weight gain (defined as a $2 \mathrm{~kg}$ increase over 48 hours or less);

2. New York Heart Association functional classes II, III, or IV; due to an exacerbation of symptoms with at least 1 class deterioration;

3. evidence of systolic and/or diastolic dysfunction on echocardiographic examination on admission (see below);

The severity of CHF was evaluated according to the medical history, New York Heart Association classification (NYHA), echocardiographic left ventricular ejection fraction (LVEF) and the determination of brain natriuretic peptide (BNP) plasma level. In the whole population the complete protocol (functional class, echocadiographic evaluation, BNP plasma level, non-invasive cardiac output and neuropsychological assessment) was obtained from 24 to 48 hours before discharging. Echocardiograms were performed with a GE Vivid 7 Pro, according to the recommendations of the American Society of Echocardiography [10]. Twodimensional apical 2- and 4-chamber views were used for volume measurements; LVEF was calculated with a modified Simpson's method using biplane apical (2- and 4chamber) views. . Left ventricular systolic dysfunction was defined as an LVEF $<50 \%$. The classification of diastolic dysfunction was a predefined modification of classifications already used [11]:

1) Impaired relaxation: early filling wave (E) /atrial filling wave (A) ratio $(\mathrm{E} / \mathrm{A})<1$ and deceleration time $(\mathrm{DT})>220$ msec in subjects aged $<55$ years or E/A $<0.8$ and DT $>220$ msec in subjects aged $>55$ years; systolic wave (S)/ diastolic wave (D) ratio of pulmonary veins (PV) $(\mathrm{PV} \mathrm{S} / \mathrm{D})>1$, and atrial reversal flow velocity $(\mathrm{AR})<35 \mathrm{~cm} / \mathrm{sec}$;

2) Pseudonormal: E/A 1 to 2, DT 150 to $220 \mathrm{msec}, \mathrm{PV}$ S/D ratio $<1$, and $\mathrm{AR}>35 \mathrm{~cm} / \mathrm{sec}$;

3) Restrictive: (E/A $>2$, DT $<150 \mathrm{msec}$, S/D ratio $<1$, and AR $>35 \mathrm{~cm} / \mathrm{sec}$ ).

In patients suffering from atrial fibrillation at the time of the echocardiogram, the diastolic function was classified as: 1) restrictive pattern (DT $\leq 150 \mathrm{msec}$ ) or 2) indeterminate (DT $>150 \mathrm{msec}$ ). The patients were also classified for left ventricular dilatation (end-diastolic diameter $\geq 57 \mathrm{~mm}$ ). The pulmonary artery pressure (PAP) was obtained by determining the peak velocity of the tricuspid regurgitation jet, adding 5 or $10 \mathrm{mmHg}$ as right atrial pressure according to right atrial size, severity of regurgitation and appearance of the inferior vena cava. From Doppler tissue imaging of the annulus the E' wave (early annular velocity opposites in direction to the mitral inflow) was determined and the ratio $\mathrm{E} / \mathrm{E}^{\prime}$ calculated [12]. The right ventricle function was investigated using the M-mode echocardiography obtaining the tricuspid annular plane systolic excursion (TAPSE) [13].

All the echo examinations were performed by expert operators blinded to the results of BNP assay; the intraobserver variability was found to be $<5 \%$. The medical therapy was normally assumed before the echocardiogram and the BNP measurement was obtained before the echo examination.

BNP was measured collecting blood sample by venopuncture and immediately analysed with the bedside Triage B type natriuretic fluorescence immunoassay (Biosite Diagnostics, La Jolla, CA, USA). The Triage Meter is used to measure BNP concentration by detecting a fluorescent emission that reproduces the amount of BNP in the blood. Two hundred and fifty $\mu \mathrm{l}$ of whole blood was added to the disposable device, next the cells were filtered and divided from the plasma with BNP, which entered a reaction chamber, containing fluorescent BNP antibodies. After a 2-min incubation, the BNP-antibody mixture migrated to an area containing immobilised antibodies and remained fixed there. The unbound fluorescent antibodies were washed away by the excess sample fluid. Then, the Triage Meter measured the fluorescent intensity of the BNP assay area. The assay results were complete in 15 minutes.

A comprehensive battery of psychological and neuropsychological tests measuring a broad range of cognitive functions was administered to each patient. The complete battery of tests consisted of the Mini-Mental State Examination (MMSE) [14], the Hospital Anxiety and Depression Scale (HADS) [15] and the Geriatric Depression Scale (GDS) [16]. All the tests have good psychometric properties and were chosen since they allow different aspects of brain functioning to be measured: cognitive and psychological functioning.

The MMSE [14] was created to allow a rapid evaluation of mental functions. This test explores two types of performances, verbal and non-verbal. In particular, the verbal subtests evaluate time and space orientation, memory, attention, recognition and concentration; the non-verbal sub-items assess the visual-motor coordination and the ability to perform a task. This instrument offers several advantages: it is easy to administrate, requires a short administration time (5 to 20 minutes), and it shows a good sensitivity in assessing a global cognitive deterioration. This test provides a score ranging from 0 to 30 ; a scoring $\geq 24$ indicates a normal cognitive performance; all scores below this cut-off generally indicate a cognitive impairment. The results of MMSE score obtained were corrected according to age and years of educational duration.

The HADS [15] is a self-assessment scale and is designed to provide a screening device for anxiety and depression in a general hospital setting. The anxiety and depression subscales are valid measures of severity of emotional disorder: for each part, a score below 8 is in the normal range, 810 is borderline and above 10 indicates a probable disorder of the relevant mood.

The 15-item GDS was commonly used to determine selfreported symptoms of depression [16]. The GDS is widely 
used and represents a robust method for assessing symptoms of depression in older population. In particular, GDS seemed to be particularly useful in ill patient population and has been used in HF populations [17].A score of 6 or greater was considered significant for the clinical presence of depression.

The recognition of depression was defined as documenttation in the medical record of any of the followings[18]: a) an outpatient diagnosis of depression described in any mental health encounters/clinic visits performed by mental health practitioners (psychiatrists) or GPs ; b) an inpatient diagnosis of depression described in any mental health encounters/clinic visits performed by mental health practitioners (psychiatrists or psychologists); c) a GDS score $\geq 6$ or an HADS $>10$.

For the measurement of non-invasive cardiac output (CO), an inert gas rebreathing method (Innocor, Innovison A/S, Odense, Denmark) was used. The system utilised a $\mathrm{N}_{2} \mathrm{O}$ (blood soluble gas) and SF6 (blood insoluble gas) enriched with $\mathrm{O}_{2}$ of $0.5 \%$ and $0.1 \%$ respectively. Tidal volume was progressively increased in the closed circuit to match the physiologic increase. Use the SF6 allowed measuring the volume of lungs, valve and rebreathing bag. $\mathrm{N}_{2} \mathrm{O}$ concentration decreases during the rebreathing manoeuvre, with a rate proportional to pulmonary blood flow. Three to four respiratory cycles were needed to obtain $\mathrm{N}_{2} \mathrm{O}$ washout. $\mathrm{Ab}$ sence of pulmonary shunt was defined as arterial $\mathrm{O}_{2}$ saturation $>98 \%$ (blood sample obtained from the arterial line). In the absence of pulmonary shunt, pulmonary blood flow $=\mathrm{CO}$. This method was proved to be closely correlated with thermodilution $(\mathrm{R}=0.93)$ and the direct Fick method $(\mathrm{R}=0.94)$ [19].

The 6MWT was performed in the discharging day according to the ATS Statement of the American Thoracic Society [20]. CHF patients able to walk underwent 6MWT if did not meet the exclusion criteria (unstable angina and myocardial infarction during the previous month, resting heart rate $>120$; systolic blood pressure $>180 \mathrm{mmHg}$ or diastolic blood pressure $>100 \mathrm{mmHg}$ ).

\section{STATISTICAL ANALYSIS}

Continuous variables were expressed as mean \pm standard deviation (SD). Categorical variables (NYHA class, presence of antidepressant therapy) were analysed using the chisquare test or Fisher's exact test. In order to determine whether BNP measurement, NYHA class or other parameters correlated independently to the different results of neuropsychological tests and cognitive function, univariate logistic regression was used. To identify the relationship between variables, a model with all the significant variables in the univariate analysis was created and analysed by logistic stepwise multivariate analysis. All probability values were two-tailed and differences were considered significant with a $p$ value $<0.05$. The 7.5 version of the SPSS software for Windows, release 12.0, SPSS Inc. Chicago USA was used.

\section{RESULTS}

Three-hundred and eighteen CHF patients were admitted to our Department (200 males) in the study period; fifteen patients (14 males) died during the hospitalization and did not perform the battery of neuropsychological tests, being excluded from the analysis. In $49(15.4 \%)$ an ICD has been previously implanted for primary prevention in CHF. The mean age was 71.6 years (range $30-89$; Table 1). The mean NYHA class at admission was $2.8 \pm 0.75$, but we included patients from NYHA class II to IV. Sixty-three (20.8\%) CHF patients were admitted in NYHA class IV and 157 (51.8\%) were evaluated in class III. The LVEF ranged from 10 to $75 \%$ and the mean LVEF was $43.2 \pm 15.5 \%$; a preserved LVEF ( $\triangle 50 \%)$ was found in $119(39.3 \%)$ CHF patients. Plasma BNP at admission was $579.8 \pm 688.3 \mathrm{pg} / \mathrm{ml}$ (range 5$5000 \mathrm{pg} / \mathrm{ml}$ ) and $6 \mathrm{minWT}$, obtained in $210(69.3 \%)$ patients in which the exercise test could be executed, was calculated in $313.5 \pm 99.3 \mathrm{~m}$ (range $60-510 \mathrm{~m}$ ). The other clinical variables were described in Table $\mathbf{1}$ and in Table 2. The therapy prescribed to those patients included angiotensin converting enzyme inhibitors (ACEI) (enalapril, ramipril) in 254 (83.4\%), angiotensin receptor blockade (candesartan, valsartan) in $57(18.1 \%)$, beta-blockers (bisoprolol or carvedilol) in 238 patients $(78.5 \%)$, digoxin in $86(28.4 \%)$, loop diuretic in 303 subjects $(100 \%)$ and low dose spironolactone in 139 $(45.9 \%)$. For beta-blockers, angiotensin converting enzyme inhibitors and angiotensin receptor blockade, the patients' maximum tolerated dose was used, after an adequate titration period. At the time of admission most subjects (203/318 $63.8 \%$ ) were in sinus rhythm. The predominant aetiology of HF was ischemic $(42.5 \%$ of subjects had a previous history of coronary artery disease). According to the current Guidelines [21], 49/318 (15.4\%) patients have been implanted with an ICD for primary prevention. The recognition of a state of depression of mood was recognized into 58/318 (18.2\%) patients and a pharmacological treatment (mainly with citalopram or escitalopram) was started or maintained. In the discharged 303 CHF patients a pathological MMSE score (considered $\leq 24)$ was determined in $29(9.6 \%)$ subjects; a GDS score suggesting a depression of mood (considered $\geq 6$ ) was

Table 1. Main Characteristics of the Population Enrolled in the Study

\begin{tabular}{|l|c|}
\hline & $\mathrm{N}=303$ \\
\hline Age & $71,6 \pm 9,9(30-89)$ \\
\hline Males & $180(59.4 \%)$ \\
\hline CAD & $129(42.5 \%)$ \\
\hline CMP & $111(36.6 \%)$ \\
\hline VALV & $60(19.8 \%)$ \\
\hline NYHA II/III/IV & $83(27.4 \%) / 157(51.8 \%) / 63(20.8 \%)$ \\
\hline Diastolic function grade 1/2/3 & $110(36,3 \%) / 62(20.4 \%) / 31(10.2 \%)$ \\
\hline ICD & $49(15.4 \%)$ \\
\hline No ICD & $269(84.6 \%)$ \\
\hline
\end{tabular}

CAD: coronary artery disease; CMP: cardiomyopathy; VALV: valvular disease; NYHA: New York Heart Association classification; ICD: implantable cardiac defibrillator. 
Table 2. Main Differences in the Variables Examined in the CHF Population Enrolled

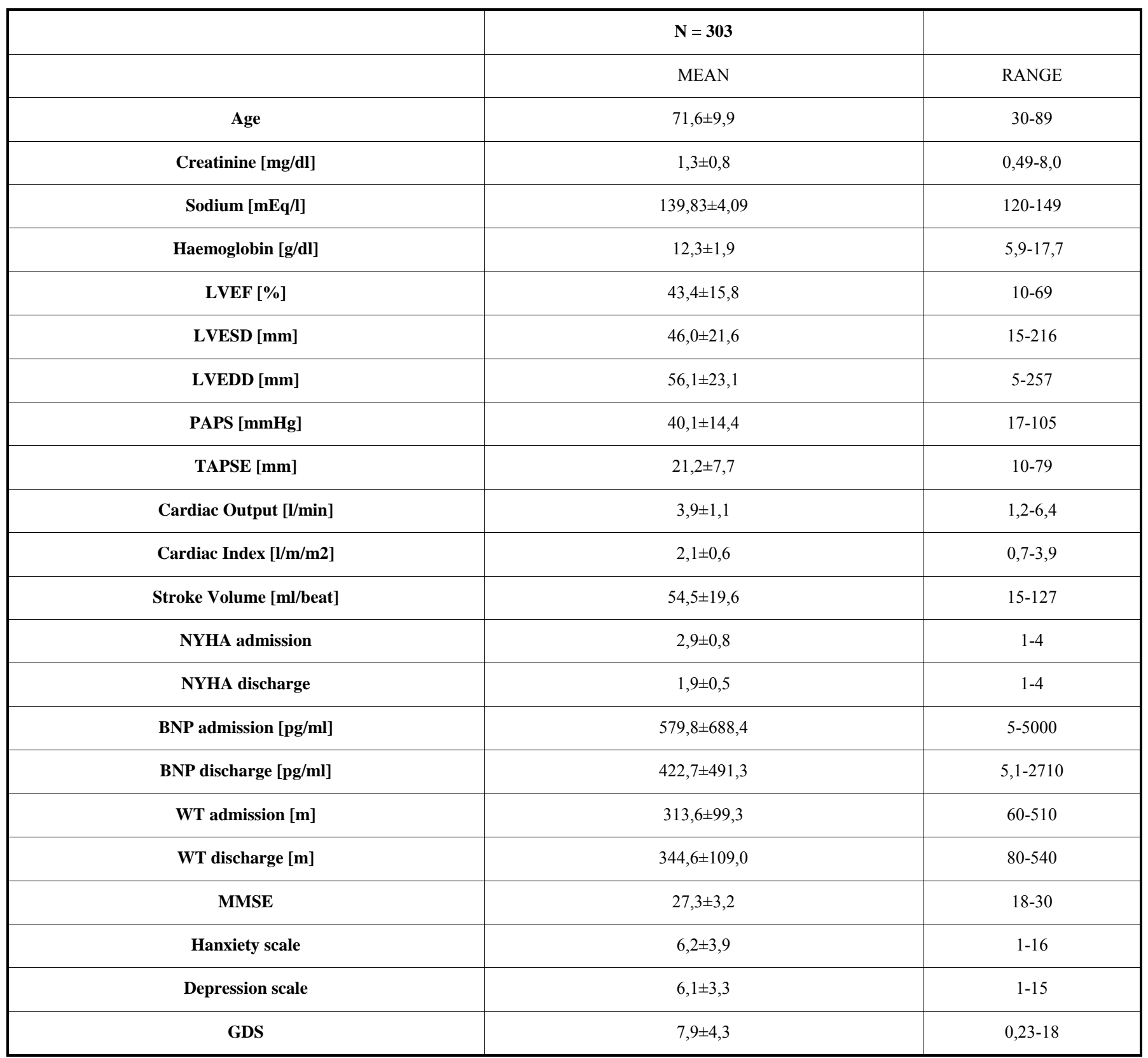

LVEF: left ventricular ejection fraction; LVESD: left ventricular end-systolic diameter; LVEDD: left ventricular end-diastolic diameter; PAP: pulmonary artery pressure; TAPSE: NYHA: New York Heart Association; BNP: brain natriuretic peptide; 6minWT: six minute walking test; MMSE: mini mental state examination; GDS: geriatric depression scale.

calculated in $23(7.6 \%)$ while an HADS score $\geq 10$ seemed to be more common (71 subjects; $23.4 \%$ ).

The univariate analysis demonstrated a positive correlation between MMSE, age $(r=0.11 \mathrm{p}=0.001)$ and BNP $(r=-$ $0.64 \mathrm{p}=0.03$ ) (Fig. 1) highlighting that CHF patients with a pathological MMSE score were older and with higher BNP plasma level. No correlations between MMSE and NYHA class $(r=0.01 \mathrm{p}=0.47)$ and $\operatorname{LVEF}(r=0.01 \mathrm{p}=0.4)$ were observed. In order to correlate the functional indexes of severity of illness, none significant relation between echocardiographic LVEF and psychological test was observed ( vs MMSE $\mathrm{p}=0.4$; vs $\mathrm{H} \mathrm{p}=0.7$; vs GDS $\mathrm{p}=0.8$ and finally vs $\mathrm{DE}$ $\mathrm{p}=0.4)$ while the NYHA class significantly correlated with the GDS ( $\mathrm{r}=0.37 \mathrm{p}=0.04)$ (Fig. 2), 6MWT with Depression scale $(\mathrm{r}=0.18 \mathrm{p}=0.01)$ with a positive trend for the noninvasive cardiac output and Depression scale $(r=0.07$ $\mathrm{p}=0.06$ ). At multivariate analysis only plasma BNP inversely correlated significantly with cognitive function (MMSE) $(\mathrm{OR}=-0.64, \mathrm{CI}=-0.42-0.86 ; \mathrm{p}=0.019)$. Finally, no significant differences in the neuropsychological performances emerged between the $214(70.6 \%)$ subjects that were discharged in sinus rhythm compared to the 89 patients in atrial fibrillation (MMSE $\mathrm{p}=0.9$; GDS $\mathrm{p}=0.16$ ). 


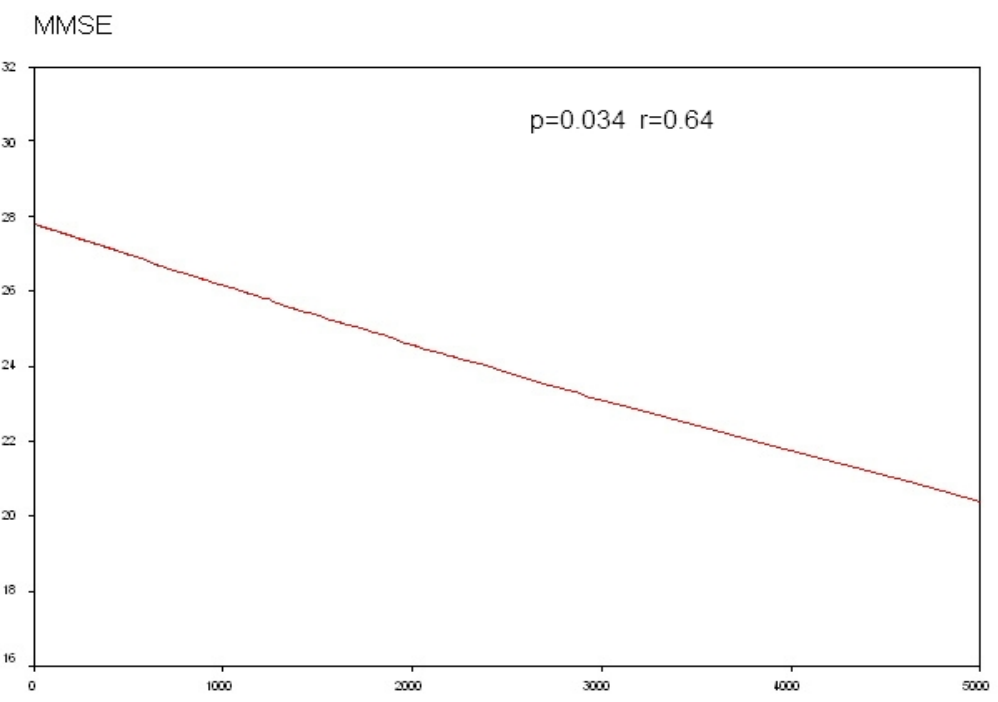

BNP

Fig. (1). Univariate analysis demonstrated a significant, inverse correlation between Mini Mental State Examination and Brain Natriuretic Peptide $(\mathrm{r}=-0.64 \mathrm{p}=0.03)$.

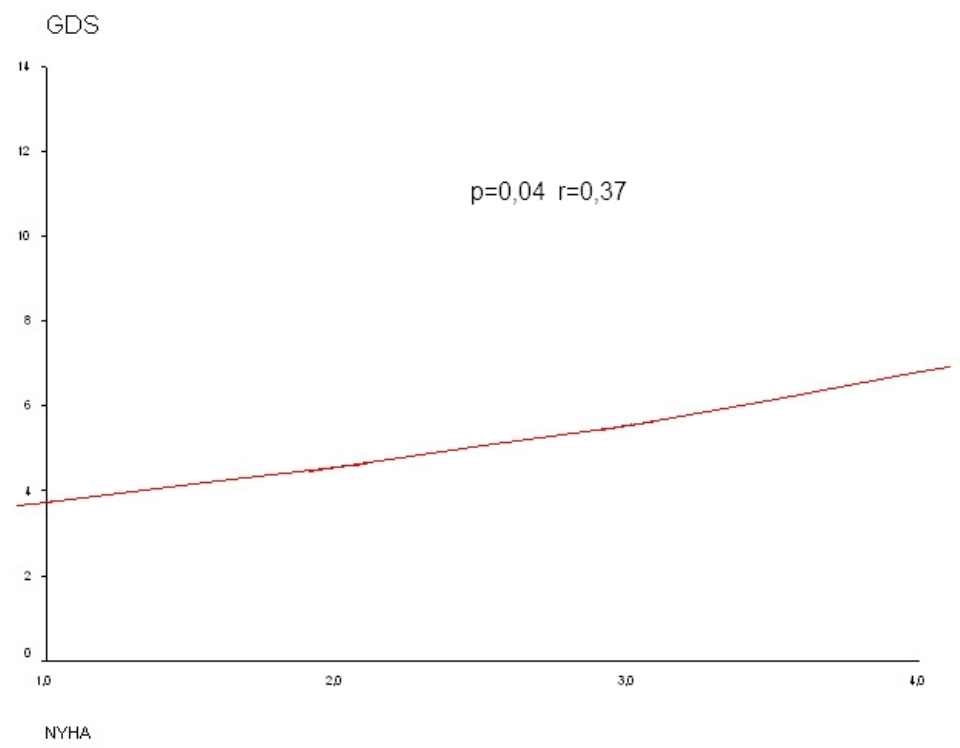

Fig. (2). In heart failure patients NYHA class significantly correlated with the Geriatric Depression Scale ( $\mathrm{r}=0.37 \mathrm{p}=0.04)$.

\section{DISCUSSION}

Cognitive impairment in patients with $\mathrm{CHF}$ is frequent and emerges through a reduction of MMSE and performance obtained in memory and learning tests. The extent of the problem is remarkably under diagnosed: it is calculated that more than $50 \%$ of patients with CHF have a MMSE scores within the dementia range (MMSE $\leq 24 / 30)$ [22-27]. The pathophysiological determinants of cognitive impairment in $\mathrm{CHF}$ patients remain uncertain. The possible mechanisms of the reduction in cognitive function in CHF patients might be simply related to pump failure [22] and cerebral hypoperfusion or even ischemic cerebral strokes [23]. Furthermore promising correlations have been found between impairment of some specific cognitive functions and different specific cardiovascular variables, without a conclusive link [22]. Indeed, the evidence that the impairment cognitive perform- ance was associated with lower indices of left ventricular function and increasing age was described [15] but the correlation between LVEF, aging and cognitive performance remains unclear. Zuccalà et al.[23]demonstrated in a large inhospital CHF population a prevalence of cognitive impairment of $35 \%$ with a positive correlation with anaemia, hypoalbuminemia, lower blood pressure levels and lower use of ACEI. Besides, CHF is associated with an increased risk of dementia and Alzheimer disease (hazard ratio of 1.84 and 1.80 respectively) and that the association between $\mathrm{CHF}$ and low diastolic pressure $(<70 \mathrm{mmHg})$ improved this risk (hazard ratio 3.07) [24]. Otherwise, different variables might play the role of confounders in the research of a link between cognitive impairment and cardiovascular parameters in $\mathrm{CHF}$ patients: a) the differences in the neuropsychological tests utilised; b) the previous history of cerebral ischemic injuries; 
c) the difficulty in evaluating the cognitive functions in patients with severe degree of CHF.

Despite the uncertainties that emerged, cognitive dysfunction in CHF subjects should be revealed because it indicates that most of the social activities are significantly impaired and, furthermore, it represents an independent prognostic marker [26-27]. In fact, in the large experience from Zuccala' et al [28] cognitive impairment was associated with a fivefold increase in mortality (relative risk of 4.9) in inhospital CHF patients. In this observational study, a severely pathological MMSE score $(\leq 24)$ was obtained in $9.6 \%$ of CHF in-patients and inversely correlated with age and plasma BNP. This correlation, obtained at univariate and multivariate analysis, confirmed, in a larger population, the results observed in a smaller population of in-hospital CHF patients [29] suggesting as BNP was a sensitive marker of cognitive dysfunction in those patients. Interesting, also in non-demented general population (313 subjects) analysed in the Hoorn study [6], plasma BNP was inversely associated with cognitive test (processing speed, memory, attention and executive function) highlighted as this biomarker might be useful for investigation as a risk factor for cognitive decline in the general population. Furthermore, the correlation between MMSE and BNP seemed to indicate that plasma BNP might play an important role not only in the prognosis of $\mathrm{CHF}$ but also as a predictive factor for recognising CHF subjects affected by cognitive impairment. Therefore the evidence of higher BNP plasma levels in patients with CHF may suggest that clinical controls should be improved in order to monitor the compliance to the prescribed therapy and to implement multidisciplinary strategies for the assessment of these subjects. MMSE and BNP plasma levels could be considered simple tests for identifying CHF patients who need special attention and systematic neuropsychological testing and require a strong doctor-patient interaction for patient's adherence to therapy.

The presence of anxiety and depression seemed to be very common in admitted or ambulatory CHF patients $[7,8]$. The recognition of anxiety and depression varied according to the differences in criteria of diagnosis; in fact, for depressive disorders may be define only by DMS-IV criteria or using neuropsychological tests (as GDS, HADS or Beck Depression Inventory). The symptomatic anxiety and depression disorders are described as under recognized and the screening of these disorders seemed to help in receiving an adequate medical treatment [8]. In particular, in OPTIMIZEHF study [30], based on 48612 in-hospital CHF patients, depression of mood has been associated with a prolonged of inhospital stay (7 vs 6.4 days in non-depressive patients, $\mathrm{p}<0.001$ ), less likely to receive coronary interventions and cardiac devices $(\mathrm{p}<0.01)$ and a worse post-discharge mortality $(\mathrm{p}=0.02)$. Our experience confirmed the strong association between the depression of mood and age $(p=0.05)$ and NYHA class $(\mathrm{p}=0.04)$ in CHF in-patients. In literature [31] a major depression disorder was more common in women, chronic pulmonary obstructive patients and severe degree of CHF (NYHA IV class). The correlation between depression of mood and NYHA class deserves two main considerations: a) the severe degree of CHF induces depression when the patient is severely functionally impaired; b) the depression might exacerbate symptoms of heart failure improving the severity of functional impairment. This latter explanation is consistent considering that a functional, objective parameters as echocardiographic LVEF [31] was not associated with depression. Our experience documented as two simple functional parameters NYHA class and 6MWT were inversely correlated to depression of $\operatorname{mood}(\mathrm{p}=0.04$ and $\mathrm{p}=0.01$, respectively), identifying a group of CHF patients in which the depressive disorder induced to report worse symptoms and functional impairment or less motivated 6MWT. The absence of a clear association between objective parameters of the clinical status in CHF (plasma BNP, LVEF and cardiac output) and the depression of mood seemed to highlight that the mood disorder might exacerbate CHF symptoms. This observation deserves to be confirmed in larger studies.

In conclusion, this study supports the necessity to evaluate the cognitive impairment and the depression of mood in in-hospital CHF patients. Cognitive dysfunction influences not only the quality of life but also the clinical evolution of the CHF compromising the doctor-patient interaction as well as patient's adherence to treatment. The results of the present study suggest that the presence of cognitive impairment in older CHF patients with higher BNP plasma level should be considered. Finally, in admitted CHF patients anxiety and depression of mood are commonly reported and influenced the perception of the severity of illness. Finally, this data confirms that a multidimensional approach is necessary to better characterize and treat CHF patients.

\section{LIMITATIONS OF THE STUDY}

The main limitation of this study is the absence of an imaging modality for analysing the cognitive impairment of CHF patients like magnetic resonance of the brain; this examination would permit to hypothesize a possible mechanism of cognitive impairment/anxiety or depression of mood in different degrees of severity of CHF.

\section{DISCLOSURES}

The authors of this manuscript have no disclosures to be declared.

\section{CONFLICT OF INTEREST}

The authors confirm that this article content has no conflicts of interest.

\section{ACKNOWLEDGEMENT}

None declared.

\section{REFERENCES}

[1] Vinson JM, Rich MW, Sperry JC, Shah AS, McNamara T. Early readmission of elderly patients with congestive heart failure. J Am Geriatr Soc 1990; 38:1290-5.

[2] Di Lenarda A, Sabbadini G, Sinagra G. Hearth Failure Programs and Comprehensive Management in Heart Failure. Monaldi Arch Chest Dis 2002; 58: 135-9.

[3] Callegari S, Majani G, Giardini A, et al. Relationship between cognitive impairment and clinical status in chronic heart failure patients. Monaldi Arch Chest Dis 2002; 58: 19-25. 
[4] Roine RO, Kajaste S, Kaste M. Neuropsychological sequelae of cardiac arrest. JAMA 1993; 269: 237-42.

[5] Farmer ME. Cognitive deficits related to major organ failure: The potential role of neuropsychological testing. Neuropsychological Rev 1994; 4: 117-60.

[6] Van den Hurk K, Reijmer YD, van den Berg E, et al. Heart Failure and cognitive function in the general population: the Hoorn Study. Eur J Heart Fail 2011; 13: 1362-9

[7] Freedland KE, Rich MW, Skala JA, et al. Prevalence of depression in hospitalized patients with congestive heart failure. Psychosom Med 2003; 65: 119-28

[8] Cully JA, Jimenez DE, Ledoux TA, Deswal A. Recognition and treatment of depression and anxiety symptoms in heart failure. Prim Care Companion. J Clin Psychiatry 2009; 11: 103-9

[9] McKee PA, Castelli WP, McNamara PM, Kannel WB. The natural history of CHF. The Framingham study. N Engl J Med 1971; 285: 1442-6.

[10] Sahn DJ, Allen HD, George W, Mason M, Goldberg SJ. The utility of contrast echocardiographic techniques in the care of critically ill infants with cardiac and pulmonary disease. Circulation 1977; 56: 959-68.

[11] Garcia MJ, Thomas JD, Klein AL. New Doppler echocardiographic applications for the study of diastolic function. J Am Coll Cardiol 1998; 32: 865-75.

[12] Gottdiener JS, Bednarz J, Devereux R. American Society of Echocardiography. American Society of Echocardiography Recommendations for use of echocardiography in clinical trials. J Am Soc Echocardiogr 2004; 17: 1086-119

[13] Lindquist P, Calcutteea A, Henein M. Echocardiography in the assessment of right heart function. Eur J Echocardiogr 2008; 9:22534

[14] Folstein MF, Folstein SE, McHugh PR. Mini-Mental State. A practical method for grading the cognitive state of patients for the clinician. J Psychiatr Res 1975; 12: 189-98.

[15] Zigmond AS, Snaith RP. The Hospital Anxiety and Depression Scale. Acta Psychiatr Scand 1983; 67: 361-70.

[16] Sheikh JI, Yesavage JA. Geriatric Depression Scale (GDS): recent evidence and development of a shorter version. Clin Gerontol 1986; 5: 165-73

[17] Vaccarino V, Kasl SV, Abramson J, et al. Depressive symptoms and risk of functional decline and death in patients with heart failure. J Am Coll Cardiol 2001; 38: 199-205

[18] Cully JA, Jimenez DE, Ledoux TA, Deswal A. Recognition and treatment of depression and anxiety symptoms in heart failure. Prim Care Companion. J Clin Psychiatry 2009; 11: 103-9.
[19] Agostoni PG, Cattadori G, Apostolo A, Contini M, Palermo P, Marenzi G, Wasserman K. Non-invasive measurement of cardiac output during exercise by inert gas rebreathing technique: a new tool for heart failure evolution. J Am Coll Cardiol 2005; 46: 1779-83

[20] ATS Statement guidelines for the six-minute walk test. ATS Committee on Proficiency Standards for Clinical Pulmonary Function Laboratories. Am J Respir Crit Care Med 2002; 166: 111-7.

[21] Epstein AE, DiMarco JP, Ellenbogen KA, et al. ACC/AHA/HRS 2008 guidelines for device-based therapy of cardiac rhythm abnormalities: executive summary: a report of the American College of Cardiology/American Heart Association Task Force on Practice Guidelines (Writing Committee to Revise the ACC/AHA/NASPE 2002 Guideline Update for Implantation of Cardiac Pacemakers and Antiarrhythmia Devices). J Am Coll Cardiol 2008; 51:e1-62.

[22] Staniforth AD, Kinnear WJM, Cowley AJ. Cognitive impairment in heart failure with Cheyne-Stokes respiration. Heart 2001; 85:1822.

[23] Zuccalà G, Cattel C, Manes-Gravina E, Di Niro MG, Cocchi A, Bernabei R. Left ventricular dysfunction: a clue to cognitive impairment in older patients with heart failure. J Neurol Neurosurg Psychiatr 1997; 63: 509-12.

[24] Zuccalà G, Marzetti E, Cesari M, et al.Correlates of cognitive impairment among patients with heart failure: results of a multicenter survey. Am J Med 2005; 118: 496-502.

[25] Qiu C, Winblad B, Marengoni A, Klarin I, Fastbom J, Fratiglioni L. Heart failure and risk of dementia and Alzheimer disease. Arch Intern Med 2006; 166: 1003-8.

[26] Del Sindaco D, Pulignano G, Di Lenarda A, et al. Role of a multidisciplinary program in improving outcomes in cognitively impaired heart failure older patients. Monaldi Arch Chest Dis 2012; 78: 20-8

[27] Dodson JA, Truong TTN, Towle VR, Kerins G. Cognitive impairment in older adults with heart failure. Prevalence, documentation, and impact on outcomes. Am J Med 2013; 126: 120-6

[28] Zuccala' G, Pedone C, Cesari M, et al. The effects of cognitive impairment on mortality among hospitalized patients with heart failure. Am J Med 2003; 115: 97-103.

[29] Feola M, Rosso GL, Peano M, et al. Correlation between cognitive impairment and prognostic parameters in patients with congestive heart failure. Arch Med Res 2007; 38: 234-9

[30] Albert NM, Fonarow GC, Abraham WT, et al. Depression and clinical outcomes in heart failure: an OPTMIZE-HF analysis. Am J Med 2009; 122: 366-73

[31] Spertus JA, McDonell M, Woodman CL, Fihn SD. Association between depression and worse disease-specific functional status in outpatients with coronary artery disease. Am J Heart 2000; 140: $105-10$

Received: May 15, 2013

Revised: 18 June, 2013

Accepted: 18 July, 2013

(C) Feola et al.; Licensee Bentham Open.

This is an open access article licensed under the terms of the Creative Commons Attribution Non-Commercial License (http://creativecommons.org/licenses/ by-nc/3.0/) which permits unrestricted, non-commercial use, distribution and reproduction in any medium, provided the work is properly cited. 